\title{
Nanobiology—Symphony of bioscience and nanoscience
}

\author{
Xian-En Zhang* \\ National Laboratory of Biomacromolecules, CAS Center for Excellence in Biomacromolecules, Institute of Biophysics, Chinese Academy of \\ Sciences, Beijing 100101, China
}

Received May 22, 2020; published online June 15, 2020

Citation: Zhang, X.E. (2020). Nanobiology—Symphony of bioscience and nanoscience. Sci China Life Sci 63, 1099-1102. https://doi.org/10.1007/s11427-020$1741-\mathrm{y}$

Nanobiology is a typical convergence science. Aiming to better understand this dynamic research field, here, we discuss the definition, scope and research trends of nanobiology.

\section{Early inspiration}

In 1959, quantum physicist Richard Feynman made a historic speech at Caltech (Feynman, 1960). He opened a question "why cannot we write the entire 24 volumes of the Encyclopedia Britannica on the head of a pin". To achieve that goal, it is necessary to reduce the size of the writing tool by 25,000 times and by that time, it seemed impossible. Then Feynman turned to the biological system: it "can be exceedingly small. Many of the cells are very tiny, but they are very active; they manufacture various substances", "they do all kinds of marvelous things - all on a very small scale", "which does what we want" to do. The question challenged generations and spawned an emerging scientific field-nanotechnology in the following decades, which might be well demonstrated by the successive advent of carbon nanomaterials, from fullerene (C-60), to carbon nanotube, to graphene, and their applications (Jariwala et al., 2013).

\section{Bio- meets nano-}

In the same era, biological science has undergone revolutionary changes, driven by the discovery of DNA double

\footnotetext{
*Corresponding author (email: zhangxe@ibp.ac.cn)
}

helix. On one hand, with the development of molecular cell biology, structural biology and bio-omics, we have an unprecedented understanding of structure and function of biomacromolecules, cells and cellular process and its regulation. The integration of biology and nanoscience revealed that a live cell is exactly a natural nano factory full of molecular machines. They work in coordination to maintain cellular processes.

On the other hand, gene technology and synthetic biology enable us to manipulate biomacromolecules or even de novo synthesize biological systems. By combined use of these biological technologies and nano characterization technology, it becomes reality to build bio-based and hybrid biological nanodevices with the properties beneficial to human beings.

Thereupon, nanobiology and nanobiotechnology as a new interdisciplinary frontier science has come into being since the turn of the 21 st century.

\section{Concept and definition}

Although many discussions about nanobiology can be found through the search engines such as Baidu and Google, it is difficult to find its definition. There are multiple reasons why it is difficult to define an emerging interdisciplinary research field. First, due to "emerging", its connotation and scope are yet to be explicated. Second, because of "interdisciplinary", the attribution of disciplines is still unclear. This situation is exactly the driving force for the development of cross-cutting science. Moreover, the term nanobiology is often used 
interchangeably with nanobiotechnology.

Based on comprehensive analysis, nanobiology may be defined as follows. Nanobiology is to understand the structure, function and operation mechanism of biological systems at the microscale from the perspective of nanoscience, to study the biological effect of nanomaterials, and to use the methods and tools of biotechnology and nanotechnology to create bio-based or bionic functional nanostructures and nanodevices so as to develop nanobiotechnology and benefit human beings. In short, nanobiology is to learn and mimic the biological systems, while nanobiotechnology is to develop and utilize these biological systems.

\section{Distinct features of the biological nanoworld}

Biological machine system has several obvious characteristics: self-assembly, high efficiency, high precision and high fidelity, flexibility, chemical energy driven, and self-regulation (Zhang, 2008).

Structural biology and cell biology have shown us the perfect combination of structure and function of biomacromolecules, microcompartments, organelles, whole cells, etc., which is a masterpiece of natural design. In addition to chemical interpretation that defines chemical force of selfassembly (e.g., van der Waals force and hydrogen bond), the self-assembly in biology is normally assisted by various molecular chaperons and post modification, and subjected to the timing control.

High efficiency of cell factories can be represented by enzymatic catalysis. The turnover number of enzyme molecules is about $10^{3}$, which is much higher than that of chemical catalysts and mimetic enzyme. DNA replication machines can synthesize 1,000 nucleotides per second in $E$. coli cells while $50 \mathrm{bp}$ per second in human cells. While to ensure the high speed of replication in humans, DNA replication is initiated at multiple sites on chromosomes.

High fidelity is critical to life processes. Again, taking DNA replication as an example, its fidelity determines the stability of genome. There are at least three lines of defense for the accuracy or fidelity of DNA replication machine: (i) base-specific pairing of DNA double strand; (ii) base pairing proofreading module of DNA polymerase; and (iii) the repair system of base mismatch in newly synthesized DNA fragments. Through these three progressive processes, the probability of a newly synthesized DNA containing wrong base is mere $10^{-8}-10^{-10}$ (Kunkel, 2004).

Cellular processes are driven by chemical energy, which comes from various carbohydrates. However, the energy contained in these nutrients cannot be directly used as cell energy. Instead, the energy released during the decomposition of the carbohydrates is stored in the form of ATP, which becomes the universal "energy currency" for all kinds of life systems.

As a nano factory, cells should have a self-regulation mechanism to control information flow, material flow and energy flow. The self-regulation includes forward feedback and backward feedback, which could be triggered by single factor and carried out directly or in cascade or even in networking.

\section{Research trends}

Natural biological nanostructures, such as molecular machines, e.g., protein synthesis machines, bacterial flagella (Johnson et al., 2020), ion channel pumps, molecular motors (rotary or linear) (Bai and Warshel, 2019; van den Heuvel and Dekker, 2007), and some artificial bio-based devices such as DNA origami (Ke et al., 2012), molecular sensors (Clapham, 2003; Zhang et al., 2016), and molecular carriers (e.g., exosomes) (Maas et al., 2017), have been extensively studied. The research focus has been the fine combination of their structure and function, the driving force of selfassembly and the regulatory mechanism.

Nano biological effect refers to the effects of special physical and chemical properties of nanomaterials on biological processes. On the one hand, it promotes the development of nano toxicology and establishes the nano biological safety evaluation system; but on the other hand, some biological effects can enhance the efficacy of drugs, such as increasing the reachability of drugs to their targets and the absorption of drugs by the human body. Some nano drugs can even overcome biological barriers (such as BBB) to reach the lesion.

Nano bionics is a very attractive domain. Inspired by nature of the biological nanoworld, scientists are keen to design and create new nanostructures for the novel or specific functions. For example, it was found that the super hydrophobic effect of the micro-nano structure on the lotus leaf surface is the root cause for the self-cleaning process (Liu and Jiang, 2011). In the study of chemically simulated biological systems, super hydrophobic surfaces exhibit a vast application prospect.

Various nano products have emerged, including nano biomaterials, nano drugs and reagents, nano biosensors, and nano biocatalysts. They are bioBricks based, or biomimetic, or hybrid of biological and physical/chemical materials. Their special properties present promising applications in life science research, medical, industrial and agricultural fields, environmental monitoring, quarantine, and antiterrorism.

\section{Topics of this issue}

Due to the limited space, six research teams have been se- 
lectively invited to contribute to the special topic of this issue. Their prospective and systematic research in respective directions have formulated distinctive features, including natural biological nanomachines and biosensors, rational design of biomacromolecules-based nano devices, biomimetic nano devices, and biological effects of nanomaterials. We expect that the collection of these papers, to some extent, reflects the representative progress in nanobiology.

Natural molecular motors exist in all kinds of organisms. They play key roles in driving the material flow and energy flow in cells. Peixuan Guo has made unremitting efforts to reveal the mechanism of molecular motors. Here, taking the viral DNA packaging motor as an example, his team describes precision motor channel, motor stoichiometry of homologous multi-subunit, and hand-in-hand integration of the hexametric pRNA ring. The authors systematically review the long-term research of molecular motor and emphasize the importance of the basic research, in particular, from understanding the operating mechanism of the molecular motor to biomedical applications (Liang et al., 2020).

The concept of nucleic acid framework was suggested by Chunhai Fan. By topology design, single-stranded DNA or RNA can be patterned by self-assembly techniques such as origami to form a variety of nanostructures, exhibiting the beauty of biological nanoart. Among them, the DNA framework structure not only has the characteristics of highprecision programmability, diversity, rigidity, etc., but also can produce specific physiological and biochemical functions. They are ideal nano building blocks. Here, the DNA framework structure-based electrochemical biosensors are reviewed as an example to demonstrate how to design and create a DNA framework according to the desired purpose (Li et al., 2020a).

Protein nano devices are also versatile. They can have onedimensional, two-dimensional, three-dimensional or hierarchical structures. Through rational design, the protein nanostructures can be reconstructed in vitro and in vivo. Studying self-assembly of protein nanostructures can not only help us to deepen our understanding of the biological machines made of the proteins (build-to-earn), but also exploit them in applications (build-to-use), such as biosensing, live cell imaging, drug delivery, and biocatalysis. The review by my colleagues Feng $\mathrm{Li}$ et al. focuses on the characteristics, functionalization strategies and applications of protein nanostructures ( $\mathrm{Li}$ et al., 2020b).

Olfactory and taste receptors are the recognition elements of typical natural biosensors, which have high selectivity for target molecules. By combining them with physical transducers and carbon nano materials such as carbon nano tubes and graphene, the sensitivity of artificial nano biosensors can be improved. I had many times enjoyed Tai Hyun park's beautiful lectures on this subject. Here, his team presents various types of olfactory/taste receptors-based nano biosensor platforms, which can be applied to a wide range of interests, including food and beverage industry, environmental monitoring, biomedical field, and anti-chemical terrorism (Moon et al., 2020).

The peroxidase-like effect (nanozyme) of nano iron-based materials discovered by Yan Xiyun's team opened a new research direction of nanomaterials with enzyme-like activity and shows great potential in biochemical detection, environmental monitoring, disease treatment and other applications due to its simple preparation and better stability than natural enzymes. However, the enzymatic properties of nanozymes need to be improved. The authors here introduce the commonly used bio-inspired strategies to design "ideal" nanozymes and discuss the progress and challenges of the strategies (Zhang et al., 2020).

The extensive application of nanobiotechnology results in a large number of nano materials entering the environment, which has increased risk of human exposure and absorption. Understanding the interaction between nano materials and biological systems is the basis of evaluating nano biological effects and toxicology. Systematic investigation of nano toxicity and effect of nanomaterials has been carried out by Chunying Chen and Yuliang Zhao's team. They suggested that the risk of exposure is related to the size of nanomaterials. This deduction is supported by in vivo distribution and toxicity of nano materials with different sizes after entering the biological system (Wang et al., 2020).

\section{Back to the original intention}

Quantum mechanics successfully depicts the random motion of atoms in the micro-world that obeys uncertainty principle. However, atoms in biomacromolecules are restricted by chemical bonds, and the 3D structure determination of biomacromolecules composed of atoms can achieve atomic or near-atomic resolution. Biomacromolecules and their complexes are nano-sized and their motion should also have thermokinetic properties. However, biomacromolecules are executors of various biological functions, their behavior is often purposeful, which is guided by the interaction with contexture molecular events and response to stimuli that change the inside and outside environment of cells. The superposition and collection of a large number of such purposeful movements, as a whole, forms an extremely complexed but highly ordered and precisely timed cycling system which accurately maintains and determines the cellular process.

In the past half century, we have established genetic central dogma, mapped numerous metabolic and regulatory pathways, acquired rich knowledge of -omics and systems biology, and comprehended more or less the cell proliferation, 
differentiation and regulation. However, we are still far away to answer what rules determine the working direction and rhythm of cells. The perfect integration of information, energy and matter flow in a living cell cannot be explained solely by the genetic central dogma and the principle of molecular interaction. This is similar to the fact that the existing physical theory cannot characterize the dark matter and dark energy of the universe. We anticipate that the indepth study of nanobiology may provide us with a new dimension to understand the fundamental principle guiding and governing cellular processes.

\section{References}

Bai, C., and Warshel, A. (2019). Revisiting the protomotive vectorial motion of $\mathrm{F}_{0}$-ATPase. Proc Natl Acad Sci USA 116, 19484-19489.

Clapham, D.E. (2003). TRP channels as cellular sensors. Nature 426, 517524.

Jariwala, D., Sangwan, V.K., Lauhon, L.J., Marks, T.J., and Hersam, M.C. (2013). Carbon nanomaterials for electronics, optoelectronics, photovoltaics, and sensing. Chem Soc Rev 42, 2824-2860.

Johnson, S., Fong, Y.H., Deme, J.C., Furlong, E.J., Kuhlen, L., and Lea, S. M. (2020). Symmetry mismatch in the MS-ring of the bacterial flagellar rotor explains the structural coordination of secretion and rotation. Nat Microbiol https://doi.org/10.1038/s41564-020-0703-3.

Ke, Y., Ong, L.L., Shih, W.M., and Yin, P. (2012). Three-dimensional structures self-assembled from DNA bricks. Science 338, 1177-1183.

Kunkel, T.A. (2004). DNA replication fidelity. J Biol Chem 279, 1689516898.

Li, F., Li, Q., Zuo, X., and Fan, C. (2020a). DNA framework-engineered electrochemical biosensors. Sci China Life Sci 63, https://doi.org/ 10.1007/s11427-019-1621-0.

Li, F., Wang, D., Zhou, J., Men, D., and Zhang, X.E. (2020b). Design and biosynthesis of functional protein nanostructures. Sci China Life Sci 63, https://doi.org/10.1007/s11427-019-1641-6.

Liang, C., Weitao T., Zhou L., and Guo P. (2020). Translation of the longterm fundamental studies on viral DNA packaging motors into nanotechnology and nanomedicine. Sci China Life Sci 63, https://doi.org/ 10.1007/s11427-020-1752-1.

Liu, K., and Jiang, L. (2011). Bio-inspired design of multiscale structures for function integration. Nano Today 6, 155-175.

Maas, S.L.N., Breakefield, X.O., and Weaver, A.M. (2017). Extracellular vesicles: unique intercellular delivery vehicles. Trends Cell Biol 27, 172-188.

Moon, D., Cha, Y.K., Kim, S.O., Cho, S., Ko, H.J., and Park, T.H. (2020). FET-based nanobiosensors for the detection of smell and taste. Sci China Life Sci 63, https://doi.org/10.1007/s11427-019-1571-8.

Feynman, R. (1960). There's plenty of room at the bottom. In Caltech's Engineering and Science Magazine Richard Feynman. Miniaturization. H.D. Gilbert, ed. (New York, NY: Reinhold Publishing Corporation).

van den Heuvel, M.G.L., and Dekker, C. (2007). Motor proteins at work for nanotechnology. Science 317, 333-336.

Wang, X., Cui, X., Zhao, Y., and Chen, C. (2020). Nano-bio interactions: the implication of size-dependent biological effects of nanomaterials. Sci China Life Sci 63, https://doi.org/10.1007/s11427-020-1725-0.

Zhang, R., Fan, K., and Yan, X. (2020). Nanozymes: created by learning from nature. Sci China Life Sci 63, https://doi.org/10.1007/s11427-0191570-7.

Zhang X.E. (2008). Cell factory and biological nanomachines (in Chinese). Chin Sci Bull 20, 364-368.

Zhang, X.E., Cui, Z., and Wang, D. (2016). Sensing of biomolecular interactions using fluorescence complementing systems in living cells. Biosens Bioelectron 76, 243-250.

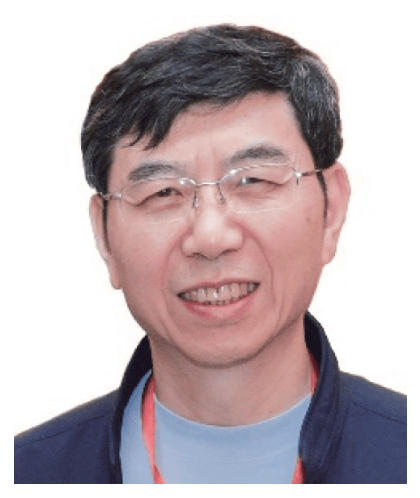

\section{Biographical Sketch}

Xian-En Zhang graduated from Hubei University in 1987 and later received MPhil (microbiology) and Ph.D. (biochemistry) from Wuhan Institute of Virology and Institute of Microbiology, Chinese Academy of Sciences (CAS), respectively. He became a full professor in Wuhan Institute of Virology, CAS in 1993. Currently he is professor of Institute of Biophysics, CAS. He has long been committed to developing biosensors, nanobiology and synthetic biology to study biological and health problems. He holds the Honorary Doctor of Science Degree granted by the University of Alberta, Canada, and is a Fellow of the Royal Society of Chemistry (RSC) and Fellow of the American Institute for Medical and Biological Engineering (AIMBE). He acts as the vice president of Chinese Society of Biotechnology, member of the permanent organizing committee of the World Congress on Biosensors, founding co-chair of the Division of Nanobiotechnology/Biosensors/Biochips, Asian Federation of Biotechnology (AFOB), and founding chair of the Biosensors/Biochips/Nanobiotechnology Committee of the Chinese Society of Biotechnology. 